\title{
Multilayer Hex-Cells: A New Class of Hex-Cell Interconnection Networks for Massively Parallel Systems
}

\author{
Mohammad Qatawneh \\ Computer Science Department, University of Jordan, Amman, Jordan \\ E-mail:mohd.qat@ju.edu.jo \\ Received September 22, 2011; revised October 13, 2011; accepted November 10, 2011
}

\begin{abstract}
Scalability is an important issue in the design of interconnection networks for massively parallel systems. In this paper a scalable class of interconnection network of Hex-Cell for massively parallel systems is introduced. It is called Multilayer Hex-Cell (MLH). A node addressing scheme and routing algorithm are also presented and discussed. An interesting feature of the proposed MLH is that it maintains a constant network degree regardless of the increase in the network size degree which facilitates modularity in building blocks of scalable systems. The new addressing node scheme makes the proposed routing algorithm simple and efficient in terms of that it needs a minimum number of calculations to reach the destination node. Moreover, the diameter of the proposed MLH is less than Hex-Cell network.
\end{abstract}

Keywords: Multilayer Hex-Cell, Interconnection Network, Parallel System

\section{Introduction}

The interconnection network strongly affects the performance of parallel machines. Many interconnection networks were proposed, from the slow but inexpensive single bus, to the extremely fast and very expensive cross-bar network [1-4]. In an interconnection network, degree related to hardware cost and diameter related to massage passing time is correlated with each other. In general, as degree of an interconnection network is increased, diameter is decreased, which can increase throughput in the interconnection network, however, it increases hardware cost with the increased number of links of the processors when parallel system is designed [2,5-7].

Among the wide variety of interconnection networks structures proposed for parallel computing systems is hex-cell which received much attention due to the attractive properties such as embeddability and simple routing [8]. However, in an identical hex-cell network the diameter increases with the increase of the network size, which can decrease throughput of the network. Ideally, we would like to construct a new network that combines features such as less communication, efficient routing and capability of embedding other static topologies. This gives the motivation to develop a new interconnection network to meet the demands of highly parallel systems.
In this paper, a new interconnection network called Multilayer Hex-Cells network (MLH), which requires less diameter than a comparable size hex-cell network and with a constant network degree, is proposed. Another advantage of the MLH network is that it is hierarchical network, which makes it suitable for the connectionist models of computations, which requires a very large number of processors and its applications are hierarchical in nature. This paper concentrates on a network that enjoys the same ease of routing as in the hex-cell network.

The organization of this paper is as follows. Section 2 describes the definition of Hex-Cell network. Section 3 introduces the proposed Multilayer Hex-Cell network. Section 4 presents the routing algorithm of MLH and Section 5 summarizes and concludes the paper.

\section{Definition of Hex-Cell Network}

A Hex-Cell network [8] with depth $\mathrm{d}$ is denoted by $\mathrm{HC}(d)$ and can be constructed by using units of hexagon cells, each of six nodes. A Hex-Cell network with depth $d$ has $\mathrm{d}$ levels numbered from 1 to $\mathrm{d}$, where, level 1 represents the innermost level corresponding to one hexagon cell. Level 2 corresponds to the six hexagon cells surrounding the hexagon at level 1 . Level 3 corresponds to the 12 hexagon cells surrounding the six hexagons at level 2 as 
shown in Figure 1. Each level $i$ has $N_{i}$ nodes, where $N_{i}$ $=6(2 i-1)$ and the total number of nodes in a Hex-Cell network $\mathrm{HC}(d)$ is $N=6 d^{2}$. Each node in the HC is identified by a pair $(X . Y)$, where $X$ denotes the line number in which the node exists, and Y denotes the location of the node in the line as in Figure 2. A node with the address 1.1 is the first node that exists at line number 1.1 .2 refers to the second node that exists at line number 1 , and so on.

\section{Multilayer Hex-Cells Interconnection Network}

The Multilayer Hex-Cell (MLH) is a modular interconnection network that consists of layers of identical Hex-Cell networks connected together in a hierarchical fashion as shown in Figure 3. The MLH is denoted by $\operatorname{MLH}(k, d)$, where $\mathrm{k}$ denotes the layer number, and $\mathrm{d}$ denotes the depth of the identical Hex-Cell. Each Layer $\mathrm{k}$ has $N_{i}$ nodes, where $N_{i}=6 d^{2}$ and the total number of nodes in MHL network is $6 \mathrm{kd}^{2}$.

\section{Lemma 1:}

The total number of nodes in a $\operatorname{MLH}(k, d)$ with $\mathrm{k}$ layers is:

$$
N_{M L H}=6 k d^{2}
$$

\section{Proof:}

Using the previous definition of $\mathrm{HC}(d)$, it can be easily seen that the total number of nodes at level 1 as shown in Figure 1 is $N_{1}=6$ nodes, since there is a single

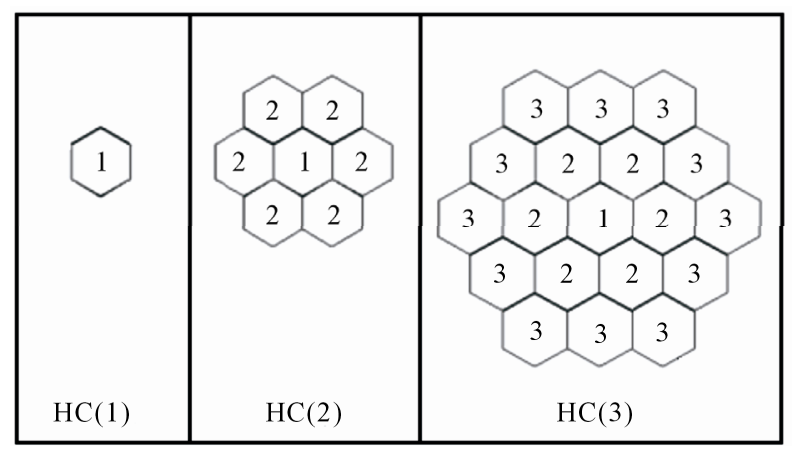

Figure 1. (a) HC (one level); (b) HC (two levels); (c) HC (three levels).

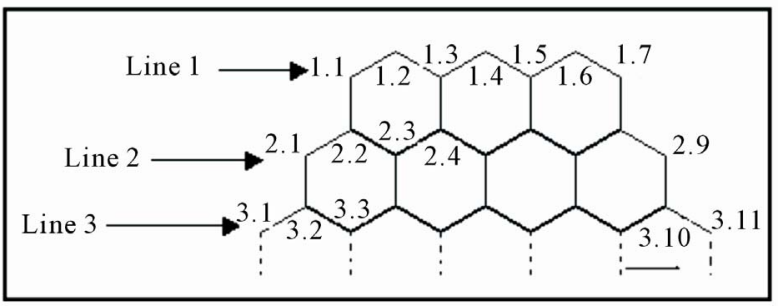

Figure 2. Addressing nodes in HC.

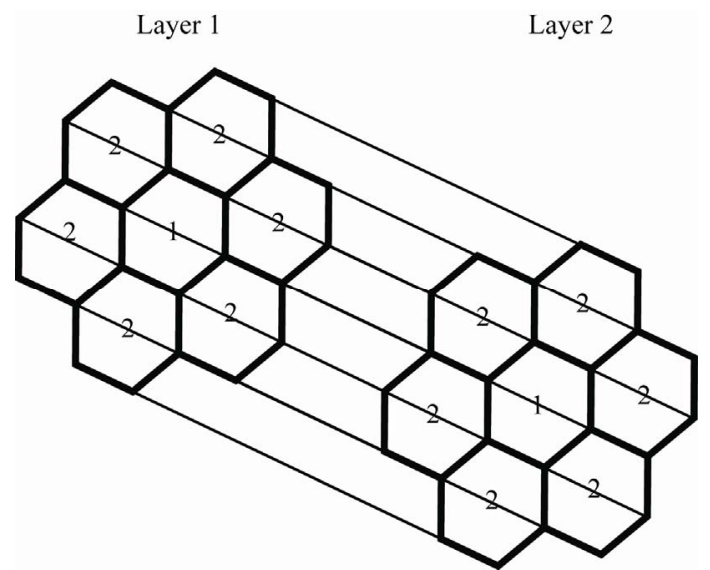

Figure 3. MLH $(2,2)$.

hexagon cell with six nodes. Level 2 introduces six hexagon cells (one at each side of the hexagon at level 1). It can be seen that level $(i+1)$ has 12 nodes in addition to corresponding nodes to those at level $i$. Therefore, the total number of nodes at level $i$ is:

$$
N_{i}=6+(i-1) * 12=6(2 i-1)
$$

The total number of nodes in an identical Hex-Cell network $\mathrm{HC}(d)$ with $d$ levels can be expresses as follows:

$$
N_{h-c}=\sum_{i=1}^{d} 6(2 i-1)
$$

Therefore,

$$
N=12 \sum i-6 \sum 1-6 d 9 d+1-6 d=6 d^{2}
$$

The total number of nodes in $\operatorname{MLH}(k, d)$ with $k$ layers can be expresses as follows:

$$
N_{M L H}=6 k d^{2} \text {. }
$$

\subsection{Node Degree}

The node degree on an interconnection network is defined as the maximum number of edges (links) that a node can have in the network. It can be easily seen that the node degree of an identical Hex-Cell with depth 1 (i.e. $\mathrm{HC}(1)$ is 2 and that for $\mathrm{HC}(d)$ is 3 , for $d>1$. Using the previous definition of $\operatorname{MLH}(k, d)$, it can be easily seen that the network degree of $\operatorname{MLH}(1,1)$ is 3 and that for $\operatorname{MLH}(k, d)$ is 4 , for $k>1$. The network degree is a desirable feature in an interconnection network that the max number of ports does not grow at the same rate as a function of the number of nodes in the network. In other words, a network topology which secures constant network degree is highly desirable. Constant node degree facilitates modularity in building blocks for scalable systems. The network degree of $\operatorname{MLH}(k, d)$ for $k>1$ is constant (4) as the network size increases. 


\subsection{Diameter}

The diameter (D) of a network is the maximum shortest path between any two nodes. If the source and destination nodes are located in the same layer (i.e. in the identical $\mathrm{HC}(d)$ the diameter is equal to $4 d-1$ [8], but if the source and destination nodes are located in different layers in $\operatorname{MLH}(k, d)$, the number of $k$ (layer) steps required to reach the destination node as shown in Case 1 and Case 2 in the proposed routing algorithm in the section 4. so the diameter of $\operatorname{MLH}(\mathrm{k}, \mathrm{d})$ is $4 d-1+k$. Figure 4 shows, that the diameter against network size of the MLH is less than Hex-Cell network.

\section{Proposed Routing Algorithm}

In order to explain the following algorithm, we use the layer and line numbering scheme as follows. Each node in the MLH is identified by a pair (K.X.Y), where $\mathrm{k}$ denotes the layer number, $X$ denotes the line number in which the node exists, and $Y$ denotes the location of the node in the line as in Figure 5. A node with the address 1.1.1 is the first node that exists at layer 1 and line number 1. The Node1.1.2 refers to the second node that exists at layer and 1 and line number 1 , and so on.

The proposed addressing node scheme can make the proposed routing algorithm simple and efficient in term of the number of operations needed to calculate the next node, especially when the source and destination nodes are located in different layers as discussed in the Examples 1 and 2. The number of calculations is equal to $n(n$ $=$ the difference between the source and destination layer numbers).

In the proposed routing algorithm for MLH as shown in Figure 6, the movement from one node to another node can be done by using one of the following three cases:

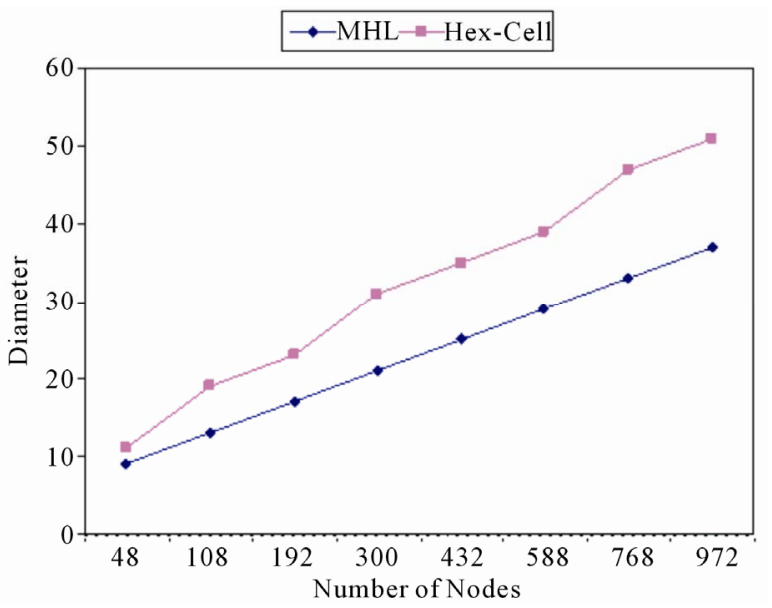

Figure 4. Diameter against the network size.

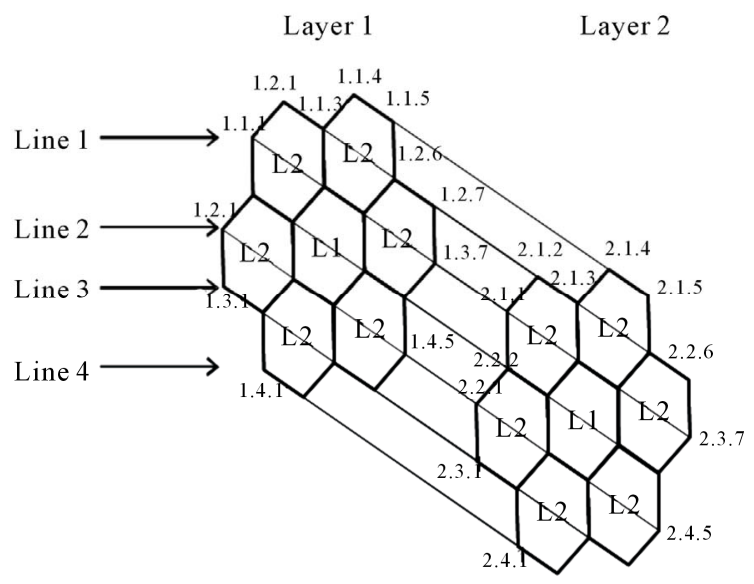

Figure 5. Addressing nodes in $\operatorname{MLH}(2,2)$.

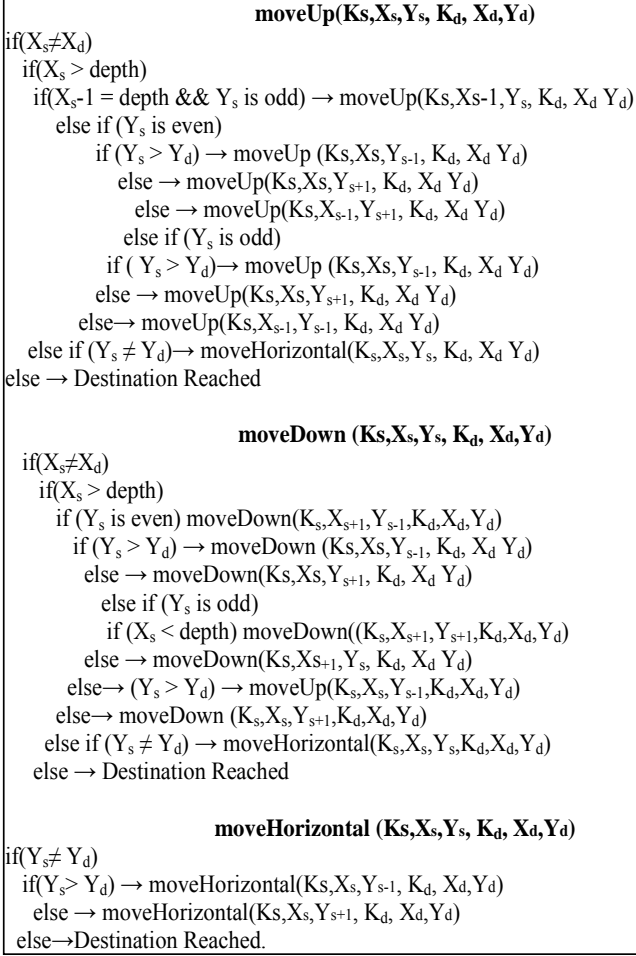

Figure 6. Routing algorithm for MLH(k,d).

CASE 1: If $\left(K_{s}=K_{d}\right) \&\left(X_{s}>X_{d}\right)$ then moveUp $\left(K_{s}, X_{s}\right.$, $\left.Y_{s}, K_{d}, X_{d}, Y_{d}\right)$

else if $\left(K_{s}=K_{d}\right) \&\left(X_{s}<X_{d}\right)$ then moveDown $\left(K_{s}, X_{s}, Y_{s}\right.$, $\left.K_{d}, X_{d}, Y_{d}\right)$

else if $\left(\left(K_{s}=K_{d}\right) \&\left(X_{s}=X_{d}\right)\right.$ then moveHorizontal $\left(K_{s}\right.$, $\left.X_{s}, Y_{s}, K_{d}, X_{d}, Y_{d}\right)$.

CASE 2: If $\left(K_{s}>K_{d}\right)$ then $\left(K_{s}-1, X s, Y s, K_{d}, X_{d}, Y_{d}\right)$.

CASE 3: If $\left(K_{s}<K_{d}\right)$ then $\left(K_{s}+1, X_{s}, Y_{s}, K_{d}, X_{d}, Y_{d}\right)$. where, $K_{s}$ is the layer number of source node, $X_{s}$ is the line number of source node, and $Y_{s}$ is the location of source node in line the number, $K_{d}$ is the layer number of destination node, $X_{d}$ is the line number of destination 
node, and $Y_{d}$ is the location of destination node in the line number. One of the previous cases will be called recursively until the destination has been reached.

\section{Discussions via Examples}

The following examples explain the above routing cases. For this purposed, we consider a network of a $\operatorname{MLH}(2,2)$ as shown in Figure 7.

Example 1: If $\left(K_{s}<K_{d}\right)$.

Let (1.1.4) and (2.2.5) be the address of the source and destination nodes respectively. CASE 3 of the routing algorithm (i.e. source layer +1 ) and moveDown will use the following path: $(2.1 .4) \rightarrow(2.1 .5) \rightarrow(2.2 .5)$ as shown in Figure 6. From Example 1, it can be easily seen that, in order to forward a message to the next node, the routing algorithm performs only one operation (subtraction) to the source layer $\left(K_{s}-1\right)$ and so on until the message reaches its destination layer. When the source and destination layers are equal, the routing algorithm forwards the message by applying the moveDown procedure as shown in the Figure 7.

Example 2: If $\left(K_{s}>K_{d}\right)$.

Let (2.3.7) and (1.1.4) be the address of the source and destination nodes respectively. CASE 2 of the routing algorithm (i.e. source layer -1 ) and moveUp will use the following path: $(1.3 .7) \rightarrow(1.2 .7) \rightarrow(1.2 .6) \rightarrow(1.1 .5) \rightarrow$ (1.1.4) as shown in Figure 6. From Example 2, it can be easily seen that, in order to forward a message to the next node, the routing algorithm performs only one operation (addition operation) to the source layer $\left(K_{s}+1\right)$ and so on until the message reaches its destination layer. When the source and destination layers are equal, the routing algorithm forwards the message by applying the moveUp procedure as shown in the Figure 7.

Example 3: If $\left(K_{s}=K_{d}\right)$.

Let (1.4.5) and (1.4.1) be the address of the source and destination nodes respectively.

CASE 1 of the routing algorithm (i.e. $K_{s}=K_{d}$ ). From the address of the source and destination nodes, it is clear that they are located at the same level (level 4). Applying the routing algorithm the moveHorizontal will be used.

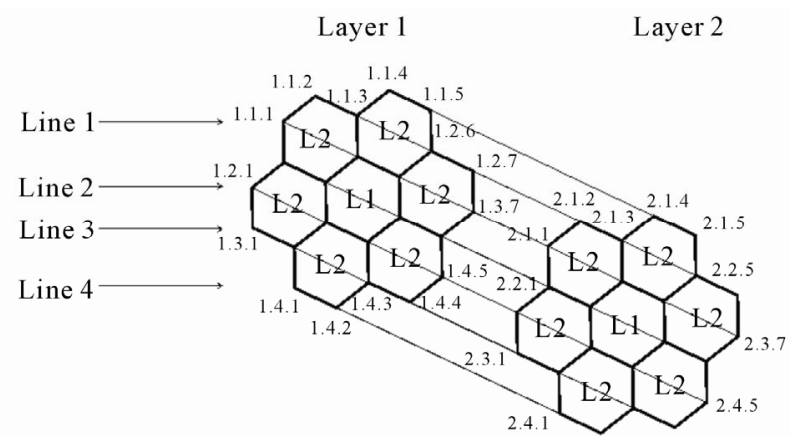

Figure 7. MLH(2,2).
The move Horizontal procedure will use the following path: $(1.4 .4) \rightarrow(1.4 .3) \rightarrow(1.4 .2) \rightarrow(1.4 .1)$.

\section{Conclusions}

This paper introduced a new interconnection topology called Multilayer Hex-Cells. Additionally, the paper proposed an efficient routing algorithm for MLH which requires no detailed knowledge of the network. The new addressing node scheme makes the proposed routing algorithm simple and efficient in terms of that it needs a minimum number of calculations to reach the destination node. The proposed MLH maintains a constant network degree regardless of the increase in the network size degree which facilitates modularity in building blocks of scalable systems. Moreover, the diameter of the proposed MLH is less than Hex-Cell.

While the proposed topology has been analyzed mathematically and discussed through examples, it can be tested as an underlining network for many applications in distributed systems area.

\section{References}

[1] A. Mokhtar, "Multi-Level Hypercube Network," Proceedings of the 5th International Conference in Parallel Processing Symposium, Anaheim, 30 April - 2 May 1991, pp. $475-480$.

[2] S. Huyn, O. Jae-Chul and L. Hyeong-Ok, "Multiple Reduced Hypercube (MRH): A New Interconnection Network Reducing Both Diameter and Edge of Hypercube," International Journal of Grid and Distributed Computing, Vol. 3, No. 1, 2010, pp. 19-30.

[3] Y. Y. Liu and J. G. Han, "Double-Loop Hypercube: A New Scalable Interconnection Network for Massively Parallel Computing. Computing," Proceedings of the ISECS International Colloquium on Computing, Communication, Control, and Management, Guangzhou, 3-4 August 2008, pp. 170-174. doi:10.1109/CCCM.2008.45

[4] T. Chan and Y. Saad, "Multigrid Algorithms on the Multiprocessor," IEEE Transactions on Computers, Vol. C-35, No. 11, 2002, pp. 969-977. doi:10.1109/TC.1986.1676698

[5] S. Syed, K. Rizvi, M. Elleithy and A. Riasat, "An Efficient Routing Algorithm for Mesh-Hypercube (M-H) Networks," Proceedings of the International Conference on Parallel and Distributed Processing Techniques and Applications, Las Vegas, 14-17 July 2008, pp. 69-75.

[6] C. Decayeux and D. Seme, "3D Hexagonal Network: Modeling, Topological Properties, Addressing Scheme, and Optimal Routing Algorithm," IEEE Transaction on Parallel and Distributed Systems, Vol. 16, No. 9, 2005, pp. 875-884. doi:10.1109/TPDS.2005.100

[7] K. Day and A. Al-Ayyoub, "Topological Properties of OTIS-Networks," IEEE Transactions on Parallel and 
Distributed Systems, Vol. 13, No. 4, 2002, pp. 359-366. doi:10.1109/71.995816

[8] A. Sharieh, M. Qatawneh, W. Almobaideen and A. Sliet, "Hex-Cell: Modeling, Topological Properties and Rout- ing Algorithm," European Journal of Scientific Research, Vol. 22, No. 2, 2008, pp. 457-468. 